\title{
Amino Acid Transporter
}

National Cancer Institute

\section{Source}

National Cancer Institute. Amino Acid Transporter. NCI Thesaurus. Code C17271.

Amino Acid Transporter proteins carry org anic compounds containing an amino (-NH2) and a carboxyl $(-\mathrm{COOH})$ group through solubility barriers, such as aqueous body fluids, lipid membranes or across epithelial layers. After binding substrate, transmembrane transporters often undergo an energy-dependent conformational change that moves the substrate across the membrane with (symport) or ag ainst (antiport) concentration gradients. 